\title{
Conducta Ortoréxica en usuarios de gimnasios de la ciudad de Cuenca
}

\section{Orthorexic Behaviour in gymns users of the city of Cuenca}

\author{
Cobos-Lazo, Felipe Andres ${ }^{1 *}$ y Hernández-Rodríguez, Yenima de la Caridad ${ }^{1}$ \\ ${ }^{1}$ Universidad Católica de Cuenca \\ *facobos101@est.ucacue.edu.ec
}

DOI: https://doi.org/10.26871/killcana_salud.v2i1.220

\begin{abstract}
Resumen
Objetivo: caracterizar la conducta ortoréxica en usuarios de dos de los gimnasios de mayor apertura y demanda de la ciudad de Cuenca, los cuales son el "Gorila Gym" y "Dorian Gym"; determinando las diferentes características sociodemográficas y sociosicológicas en relación con dicha problemática e identificando las manifestaciones y asociaciones de la ortorexia con dichos supuestos en la muestra seleccionada. Materiales y métodos: la investigación obedece a un enfoque cuantitativo, descriptivo y transversal, empleando un muestreo no probabilístico intencional de cien personas que se ejercitan diariamente en los gimnasios mencionados, con una edad promedio entre dieciséis y cuarenta seis años. Los datos fueron procesados empleando el paquete estadístico Statistical Package for the Social Sciences (SPSS) versión 23.0, para utilizar análisis de frecuencia, medidas de tendencia central, de dispersión y de asociación. El instrumento utilizado de propiedades psicométricas y que fue analizado a través de la muestra de estudio se conoce como "ORTHO-15", cuyo resultado determina la conducta ortoréxica y se compone de 15 ítems de comprensión sencilla, con un puntaje entre uno y cuatro por pregunta. Resultados: finalmente se sugiere emplear el instrumento en otras poblaciones de la región con el propósito de ratificar la presencia de ortorexia e informar de esta condición. Conclusiones: se concluyó que el 58,5\% de los usuarios de gimnasios con conducta ortoréxica se encuentran en diferentes rangos de edades, y en lo que se refiere un rango de edad de 20 a 30 años (Juventud), seguido del rango comprendido entre 18 a 20 años (Adolescente tardío) con el $27 \%$, y en lo que se refiere al género, fueron los individuos de índole masculino los que alcanzaron la mayor prevalencia de conducta ortoréxica
\end{abstract}

Palabras clave: ortorexia, hábitos alimenticios, usuarios de gimnasios, Ortho-15.

\begin{abstract}
Objective: The purpose of this research is to determine the orthorexic behavior of users of "Gorila Gym" and "Dorian Gym", two of the most popular gymnasiums in the city of Cuenca. This will be done in order to define the different socio-demographic and socio-psychological characteristics of these problems and to identify the signs and the ways in which orthorexia is related to these assumptions in the selected sample. Materials and methods: The research follows a quantitative, descriptive and cross-sectional approach, it uses an intentional non-probabilistic sampling of one hundred people who exercise daily in the aforementioned gyms, and whose ages range between sixteen and forty-six years old. The data were processed using the Statistical Package for the Social Sciences (SPSS), version 23.0, intended to use frequency analysis, central trend dispersion, and association measurements. The psychometric properties tool that was analyzed in the sample is known as "ORTHO-15", and its result identifies orthodexic behavior. It consists of 15 items of easy comprehension, with one to four marks per question. Results: Finally, the use of this tool has been suggested in other populations of the region so as to ratify the presence of orthorexia and to report this condition. Conclusions: it was concluded that $58.5 \%$ of users of gyms with orthorexic behavior are in different age ranges, and in what refers an age range of 20 to 30 years (Youth), followed by the range included between 18 to 20 years (late adolescent) with $27 \%$, and in what refers to gender, were the individuals of masculine nature those who reached the highest prevalence of orthorexic behavior
\end{abstract}

Key words: orthorexia, eating habits, gyms users, Ortho - 15.

\section{Introducción}

La ortorexia es el nuevo trastorno alimenticio del siglo vigente, que ha iniciado su incremento y presencia en el desarrollo del ser humano de una manera rápida y efi- caz. Esta problemática fue descrita por primera ocasión por el doctor estadounidense Steve Bratman ${ }^{1}$ en el año 1996, quien elaboró un autodiagnóstico de diez ítems, basándose en los síntomas y malestares que él padeció cuando era nutricionista y se regía bajo dietas extremas, 
para determinar si el individuo padece esta condición. La definición principal de ortorexia proviene del griego Ortho que significa recto, y Orexia que se refiere al apetito; por lo tanto, ortorexia vendría a definirse como "apetito justo o correcto". ${ }^{2}$ Dentro del campo de la salud física y mental, la ortorexia lleva un significado mucho más complejo y preocupante, pues el individuo que la posee sufre de una obsesión intensa por alimentarse con comida sana, que llega a un nivel que se considera patológico debido a que se enfoca en el hábito de la alimentación que generar un deterioro en otras areas de su vida personal como la familiar o laboral. ${ }^{3}$

A todo esto, se incluye la realidad de que se vive en un ambiente en donde la figura esbelta, la imagen corporal radiante y las múltiples dietas forman parte de la rutina del ser humano; por consiguiente, y aunque la ortorexia no ha sido aún registrada en los guiones terapéuticos de trastornos mentales como el DSM, el poco conocimiento que tiene la humanidad y las escasas investigaciones sobre la ortorexia, están comprobando, que debajo de la obsesión por el consumo intenso y exigente de alimentos que estén limpios de componentes artificiales y supersticiones, se esconde en la mayoría de ocasiones un trastorno mental. ${ }^{4}$

En este contexto, surgió la presente investigación en la provincia del Azuay, Ecuador y que tuvo por objetivo caracterizar la conducta ortoréxica en usuarios de los gimnasios "Gorila Gym" y "Dorian Gym" de la ciudad de Cuenca; al igual que determinar las características sociodemográficas y sociosicológicas e identificando factores y asociaciones de esta problemática con dichas variables.

\section{Marco Teórico}

La ortorexia desde el aspecto médico posee ciertos criterios diagnósticos. Entre esos supuestos, se encuentran: dedicar más de tres horas al día a pensar en su dieta sana, preocuparse más por la calidad de los alimentos que del placer de consumirlos, disminución de su calidad de vida, ${ }^{5}$ sentimientos de culpabilidad cuando no cumple el régimen dietético, planificación excesiva de lo que comerá al día siguiente y aislamiento social provocado por su tipo de alimentación. $^{6}$

Entre las personas vulnerables de padecer ortorexia, un gran porcentaje se puede observar en el adolescente y el adulto joven, quienes se encuentran presionados por la cantidad de propagandas sobre el control de peso y la belleza física $^{7}$ además de aquellos que practican deportes como el físicoculturismo o el atletismo. Un cuerpo saludable, puede arruinarse radicalmente, cuando el individuo solo se centra en su salud física, mientras su mente se estropea con pensamientos nocivos. ${ }^{8,9}$

Al igual que otro trastorno alimenticio, el tratamiento contra la ortorexia se enfoca en el trabajo interdisciplinario eficaz y preparado. Además, si se valoran los tratamientos realizados en este tipo de conductas, un enfoque cognitivoconductual sería uno de los más relevantes ya que se enfoca en los pensamientos irracionales para cambiar una conducta inadecuada. $^{10}$

Entre las investigaciones que se han desarrollado durante la última década para tener una visión más centralizada sobre esta patología, las más importantes son: en la región de la Araucanía, ciudad de Temuco, en Chile se realizó una indagación a estudiantes de una edad entre 16 y 18 años, dando como resultados que el $30.7 \%$ de los estudiantes entrevistados poseían conducta ortoréxica y que los varones fueron los que alcanzaron la mayor prevalencia con $35.1 \%$ frente a las mujeres con $25.5 \% .^{11}$ De igual manera, en la ciudad de Erzurum en Turquía realizaron una investigación sobre centrada la conducta alimentaria en 878 estudiantes que seguían la carrera de medicina donde 464 (52.8\%) eran de sexo masculino y $359(40.9 \%)$ de sexo femenino y entregando como resultados que los varones presentaban mayor prevalencia de conducta ortoréxica con un $12 \%$ frente a un $8 \%$ de las mujeres. ${ }^{12}$

En la ciudad de Saô Paulo, Brasil ${ }^{13}$ se ejecutó una exploración científica sobre la ortorexia en 392 personas que se encontraban en un régimen dietético con una edad entre treinta a cincuenta años y con un $95 \%$ de la muestra de sexo femenino. El $15 \%$ de la muestra presentó conducta ortoréxica. Finalmente, en la ciudad de Cuenca, Ecuador, en 204 participantes; 113 mujeres $(55,4 \%)$ y 91 hombres (44.6\%); de los cuales el $80,9 \%$ se encontraban dentro de los parámetros normales referente a su masa corporal; se concluyó que la prevalencia encontrada para ortorexia fue del $72.5 \%$; específicamente el $56.1 \%$ en mujeres y el $43.9 \%$ en hombres. ${ }^{14}$

Bajo todos estos parámetros yace la actual investigación debido a que se amerita comprobar o no en los gimnasios más afluentes de la ciudad de Cuenca, en usuarios y entrenadores, la existencia de un exceso de control en el consumo de calorías, una intensa presión en conseguir metas sobre su apariencia física, problemas de autoestima si rompen rutinas de ejercicios, uso de dietas estrictas y extensos gastos económicos en artículos y proteínas para conseguir un cuerpo esbelto. Todas estas características se encuentran en la calificación del instrumento Ortho-15; por ende, es relevante y necesario determinar si la ortorexia se manifiesta en estos individuos que se ejercitan a diario tanto de una manera profesional como amateur.

\section{Metodología}

La investigación obedeció a un enfoque de investigación de tipo cuantitativo, descriptivo y transversal, utilizando un muestreo no probabilístico intencional de voluntarios. La muestra investigada estuvo compuesta por usuarios que se ejercitan diariamente en los Gimnasios "Gorila" y "Dorian Gym" de la ciudad de Cuenca $(n=100)$, con alrededor de 400 asiduos anuales; los mismos que se enfocan en mantener una imagen corporal saludable y por ende la alimentación sana forma parte de su rutina diaria. El rango de edad está comprendido de 16 a 46 años, y está conformado por 47 mujeres y 53 hombres. 
Se empleó el instrumento "ORTHO-15" creado por el italiano L.M. Donini ${ }^{10}$ y validado en una muestra de 525 sujetos de distintas ciudades de Italia con una puntuación de 0,75 según la escala Alpha de Cronbach. El objetivo de este reactivo es determinar la conducta ortoréxica y se compone de 15 ítems de comprensión sencilla, con un puntaje entre uno y cuatro por pregunta; posee tres factores estructurales que son el racional sobre una valoración de 24 puntos, emocional con 16 puntos y conductual con 16 puntos. ${ }^{15,16}$ Los resultados alcanzados se catalogaron en función del puntaje conseguido en la adición de las respuestas realizadas por los encuestados voluntarios, dividiéndose en 3 categorías: conducta ortoréxica (15-35 puntos), normal (36-38 puntos) y saludable (39-60 puntos). ${ }^{17,18}$ De igual manera, cada ítem del reactivo tiene una calificación mínima de 1 punto cuando se aproxima más a una conducta ortoréxica y una calificación máxima de 4 cuando es una respuesta referente a una conducta saludable.

Los datos fueron procesados a través del programa digital Statistical Package for the Social Sciences (SPSS) versión 23.0, a través de análisis de frecuencia, medidas de tendencia central, de dispersión y de asociación. Los sujetos encuestados recibieron un consentimiento informado y una hoja de registro como evidencia para la presente investigación.

\section{Resultados}

A través de esta investigación en cien personas que se ejercitan diariamente en uno de los gimnasios existentes de la ciudad de Cuenca, se pudo evidenciar la presencia de conducta ortoréxica en un $22 \%$; siendo el sexo masculino y la edad comprendida entre 20 y 30 años las variables sociodemográficas de mayor frecuencia con 13 individuos en ambas variables. De igual manera, se concluyó dentro de las particularidades sociosicológicas, que no existen deportistas élites con conducta ortoréxica dentro de la muestra seleccionada y que 20 usuarios de gimnasios no han padecido algún trastorno alimenticio previo a la ortorexia. Finalmente, se definió que hubo una mayor dispersión en el factor estructural Racional del reactivo Ortho - 15 debido a que se obtuvo una media de 14,21 y que la fiabilidad de este instrumento a través del coeficiente de Alpha de Cronbach, es aceptable debido a que su puntuación fue de 0,748 sobre 1 .

A continuación, se detallan los resultados obtenidos en la presente investigación.

Tabla 1. Categorías de conducta ortoréxica en usuarios de gimnasios de la ciudad de Cuenca

\begin{tabular}{|c|c|c|}
\hline Categorías & $\begin{array}{c}\text { Frecuencia } \\
\text { Absoluta }\end{array}$ & $\begin{array}{c}\text { Frecuencia } \\
\text { Relativa }\end{array}$ \\
\hline Ortoréxica & 22 & 22 \\
\hline Normal & 37 & 37 \\
\hline Saludable & 41 & 41 \\
\hline Total & $\mathbf{1 0 0}$ & $\mathbf{1 0 0}$ \\
\hline
\end{tabular}

Fuente: Elaboración propia de los autores
El $22 \%$ de la muestra aplicada posee conducta ortoréxica, el $37 \%$ está en riesgo de poseer ortorexia y el $41 \%$ restante presenta una conducta saludable frente al consumo de alimentos. 
Fig. 1. Categorías de conducta ortoréxica en usuarios de gimnasios de la ciudad de Cuenca según Edad y Género

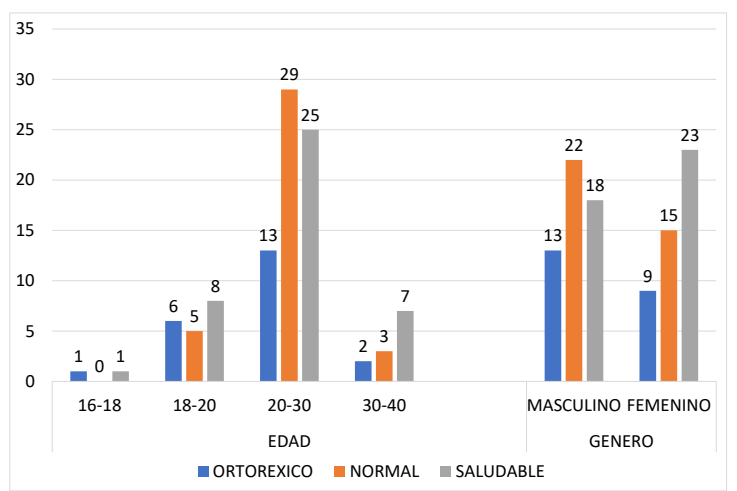

Fuente: Elaboración propia de los autores

El $13 \%$ de la muestra con conducta ortoréxica, se encuentra en un rango de edad de 20 a 30 años (Juventud), seguido del rango comprendido entre 18 a 20 años (Adolescente tardío) con el $6 \%$. De igual manera, se observa que los individuos de género masculino fueron los que alcanzaron la mayor prevalencia de conducta ortoréxica con el $13 \%$ frente a las mujeres con el $9 \%$.

Fig. 2. Categorías de conducta ortoréxica en usuarios de gimnasios de la ciudad de Cuenca según instrucción académica, procedencia y deportes practicados.

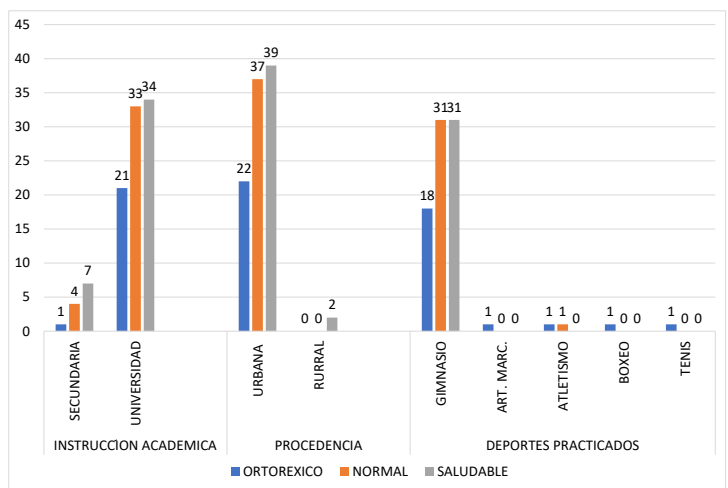

Fuente: Elaboración propia de los autores

En lo referente a la instrucción académica, el $88 \%$ de la muestra se encuentra en instrucción de tercer nivel y el $21 \%$ de los encuestados poseen conducta ortoréxica. En lo relacionado a procedencia, el $98 \%$ de los usuarios proceden de un sector urbano, en donde el $22 \%$ tiene ortorexia; y finalmente, en lo que se refiere a deportes practicados, el $4 \%$ de la población encuestada que posee conducta ortoréxica, se ejercita en un gimnasio y practica otros deportes como las artes marciales, el boxeo, el atletismo y el tenis.
Fig. 3. Categorías de conducta ortoréxica en usuarios de gimnasios de la ciudad de Cuenca según deportistas con entrenamiento élite y usuarios que han padecido un trastorno alimenticio.

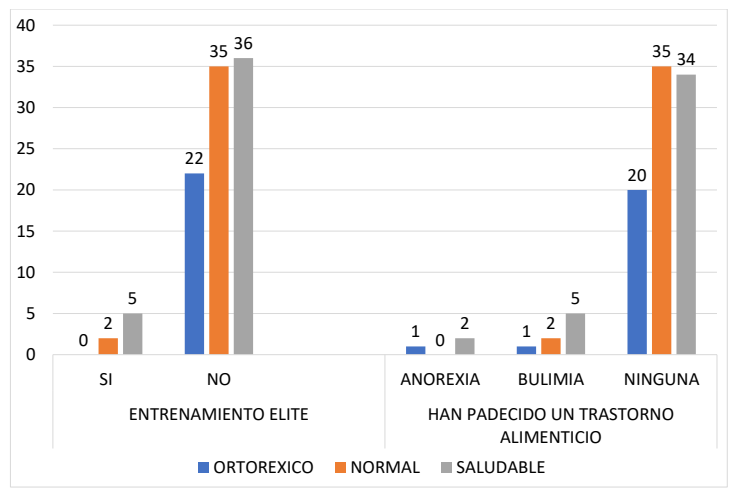

Fuente: Elaboración propia de los autores

En lo relacionado a deportistas con entrenamiento élite, en el $100 \%$ de la muestra aplicada, ninguna persona que se considera deportista élite posee conducta ortoréxica. Con respecto a usuarios de gimnasios que han padecido un trastorno alimenticio se indica que el $2 \%$ de encuestados aparte de ortorexia, también toleraron anorexia o bulimia respectivamente.

Tabla 2. Factores estructurales Racional, Emocional y Conductual en usuarios de gimnasios de la ciudad de Cuenca

\begin{tabular}{|c|c|c|c|c|}
\hline Indicador & Mínimo & Máximo & Media & Desviación \\
\hline F. Racional & 7 & 19 & 14,21 & 2,34 \\
\hline F. Emocional & 6 & 15 & 12 & 1,6 \\
\hline F. Conductual & 4 & 13 & 9,25 & 1,61 \\
\hline
\end{tabular}

Fuente: Elaboración propia de los autores

En la muestra aplicada de los usuarios de gimnasios de la ciudad de Cuenca, como dato más relevante que existe una mayor dispersión en el factor estructural Racional debido a que se obtuvo una media de 14,21 y una desviación de 2,34. Como datos adicionales se puede observar una mayor homogeneidad entre el factor estructural emocional con una media de 12 y una desviación de 1,6 y el factor estructural conductual con una media de 9,25 y una desviación de 1,61.

Al relacionar los factores de la conducta ortoréxica y las variables sociodemográficas, se determina asociación estadística débil entre el género y el factor racional, para valores de eta $=0,15$; el factor emocional puede explicarse por el género en el $34 \%$ de los casos (eta=0,34). El resto de las variables contempladas en el estudio no arroja asociación estadística con los factores de la conducta ortoréxica.

Tabla 3. Alpha de Cronbach ortho- 15

\begin{tabular}{|c|c|c|}
\hline Instrumento & $\begin{array}{c}\text { Alpha de } \\
\text { Cronbach }\end{array}$ & Descripción \\
\hline Ortho -15 & 0,748 & Aceptable \\
\hline
\end{tabular}

Fuente: Elaboración propia de los autore 


\section{Conclusiones y Recomendaciones}

Es evidente que la conducta ortoréxica en Ecuador, no es una temática que se analice a menudo y eso se refleja en las pocas investigaciones que constan y que permitan un estudio profundo de la ortorexia. Por eso, el presente trabajo se apoya en otras indagaciones extranjeras.

En primera instancia, en esta investigación se concluyó que de los 100 usuarios de gimnasios encuestados, el $22 \%$ presenta conducta ortoréxica, el $37 \%$ exhiben una conducta normal; es decir, se encontraban en riesgo de padecer ortorexia y el $41 \%$ presentaron una conducta saludable; equivalente a la exploración estadística realizada en la ciudad de Saô Paulo sobre la ortorexia en 392 personas que se encontraban en un régimen dietético con una edad entre treinta a cincuenta años; obteniendo que el $15 \%$ de la muestra presentaba conducta ortoréxica. ${ }^{13}$

En relación a los datos obtenidos, no hay averiguaciones de la conducta ortoréxica enfocadas en usuarios de gimnasios; sin embargo, se apreciaron variables sociodemográficas como la edad y el género. Dicho esto, se concluyó que el $58,5 \%$ de los usuarios de gimnasios con conducta ortoréxica se encuentran en diferentes rangos de edades, y en lo que se refiere un rango de edad de 20 a 30 años (Juventud), seguido del rango comprendido entre 18 a 20 años (Adolescente tardío) con el $27 \%$, y en lo que se refiere al género, fueron los individuos de índole masculino los que alcanzaron la mayor prevalencia de conducta ortoréxica con el $24,5 \%$ frente a las mujeres con el $19,1 \%$.

Además, se identificaron en la presente investigación datos interesantes para futuras investigaciones sobre esta condición, como por ejemplo que el 95,5\% de las personas con ortorexia se encontraban estudiando o ya lo hicieron en una institución de tercer nivel; que todos los usuarios con ortorexia provienen del sector urbano; que existe conducta ortoréxica en otros deportes como las artes marciales, el atletismo, el boxeo y el tenis, que el $9 \%$ de las personas con ortorexia también padecieron de un trastorno alimenticio aceptado en el DSM como la anorexia y la bulimia y que los deportistas élites no poseen conducta ortoréxica; siendo este dato de gran interés ya que podría denotar la preparación psicológica que posee un deportista élite de un amateur para no permitir que el ejercicio físico y el control de ingesta de alimentos de forma estricta se transforme en una problemática en la salud mental y física del individuo. ${ }^{19}$

Toda esta información tiene varios detonantes y una justificación que manifestaría la presencia de este fenómeno, se concentra en las extensas cantidades de información provenientes de los distintos medios de comunicación como radio y televisión que atacan sobre todo a adolescentes y adultos jóvenes en la permanencia de poseer un cuerpo esbelto y dejando a un lado la buena alimentación; ${ }^{9}$ generando la concepción de juicios en base a pensamientos y cogniciones distorsionados; tal y como se evidencia en la dispersión encontrada en la asociación Eta con el factor estructural del reactivo Ortho-15 que obtuvo una media de 14,21 y una desviación de 2,34. Además la presente investigación tuvo el limitante de trabajar en un muestreo no probabilístico intencional de voluntarios.

Con todo esto, se podría evidenciar que la conducta ortoréxica está tornándose en un viable problema de salud tanto físico como emocional. Es indispensable realizar más estudios pertinentes al caso y así comprobar la existencia de un nuevo trastorno mental o si se refiere a un dilema social del siglo vigente etiquetado en el género masculino y a una edad comprendida en la adolescencia tardía y en la juventud. De igual manera, con el desarrollo de nuevos trabajos de indagación sobre esta conducta, se podría definir la influencia que tienen los factores estructurales de la ortorexia en las diferentes características sociodemográficas y sociosicológicas de la población

Ante esto, se recomienda aplicar el instrumento en distintas urbes y localidades más numerosas; señalando que la fiabilidad del instrumento a través del Alpha de Cronbach ha sido categorizada como aceptable con un puntaje de .748. De igual manera, si bien el Ortho- 15 ha sido utilizado en varias investigaciones en varios países; aún no ha sido aceptado como un reactivo psicométrico en el Ecuador para su utilización en este medio; por eso, se recomienda la validación o elaboración de un reactivo que sea aprobado y sea viable en el diagnóstico de la presente anomalía.

Finalmente, la ortorexia está avanzando de manera acelerada en la destrucción del bienestar del ser humano hasta llegar al punto de ser considerada una perturbación alimenticia enfocada en la obsesión por el control de la comida. ${ }^{2}$ Sin embargo, es relevante indicar, que no existen investigaciones suficientes que ofrezcan sostén a este constructo teórico, al igual que evalúen fiabilidad en instrumentos de evaluación como el Ortho-15. ${ }^{19}$

\section{Referencias Bibliográficas}

1. Arusoğlu G, Kabakci E, Köksal G, Merdol T. Orthorexia Nervosa and Adapta on of ORTO-11 into Turkish. Turkish journal of psychiatry. 2008;19:283-291.

2. Bartrina JA. Ortorexia o la obsesión por la dieta saludable. Archivos latinoamericanos de nutrición. 2007;57:313.

3. Cavero V, Lodwing C, Lulli G, Tejada R. La ortorexia: Un nuevo reto para los profesionales de la salud. Archivos Latinoamericanos de Nutrición. 2015;65:199.

4. Donini M, Marsili D, Graziani M, Imbriale M, Canella C. Orthorexia nervosa: validation of a diagnosis questionnaire. Eating and Weight Disorders-Studies on Anorexia, Bulimia and Obesity. 2005;10:28-32.

5. Durán S, Valdés P, Godoy A, Herrera T. Hábitos alimentarios y condición física en estudiantes de pedagogía en educación física. Rev Chil Nutr. 2014;1:251.

6. Eriksson L, Baigi A, Marklund B, Lindgren E. Social physique anxiety and sociocultural attitudes toward appearance impact on orthorexia test in fitness participants. Scandinavian journal of medicine \& science in sports. 2008;18:389-394.

7. García F. Ortorexia: la obsesión por los alimentos naturales. Revista de Libros. 2008;96:26-27. 
8. García A, Ramírez I, Ceballos G, Méndez E. ¿Qué sabe Ud. Acerca de... Ortorexia? Revista mexicana de ciencias farmacéuticas. 2014;45:84-87.

9. Muñoz R, Martínez A. Ortorexia y vigorexia: ¿ nuevos trastornos de la conducta alimentaria. Trastornos de la 18 . conducta alimentaria. 2007;5:457-482.

10. Koven N, Abry AW. The clinical basis of orthorexia nervosa: emerging perspectives. Neuropsychiatric disease and treatment. 2015;p. 11: 385.

11. Jerez T, Lagos R, Valdés P, Pacheco E, Pérez C. Pre- 19. valencia de conducta ortoréxica en estudiantes de educación media de Temuco. Revista chilena de nutrición. 2015;42:41-44.

12. Fidan T, Ertekin V, Isikay S, Kirpinar I. Prevalence of orthorexia among medical students in Erzurum. Comprehensive psychiatry. 2010;51:49-54.

13. Kinzl J, Hauer K, Traweger C, Kiefer I. Orthorexia nervosa in Brazilian dieticians. Psychotherapy and psychosomatics. 2006;75.

14. Merchán G, Terreros C. Prevalencia de ortorexia, vigorexia y factores asociados en estudiantes de la Escuela de Medicina de la Universidad de Cuenca.; 2016.

15. Ringer F, Crittenden PM. Eating disorders and attachment: The effects of hidden family processes on eating disorders. European Eating Disorders Review. 2007;15:119-130.

16. Rodríguez L, Moreno S. Psicofisiología del ansia por la comida y la bulimia nerviosa. Clínica y Salud. 2007;18:99-118.

17. Sánchez FG, Rial BR. Orthorexia nervosa. A new eating behavior disorder? Actas Esp Psiquiatr. 2005;33:66-68.

8. Segura C, Ramacciotti C, Rania M, Aloi M, Caroleo M, Bruni A, et al. The prevalence of orthorexia nervosa among eating disorder patients after treatment. Eating and Weight Disorders-Studies on Anorexia, Bulimia and Obesity. 2015;20:161-166.

. Powers P, Santana C. Eating disorders. A guide for the primary care physician. Primary Care; Clin Office Practice. 2007;18.
Recibido: 23 de febrero de 2018

Aceptado: 12 de junio de 2018 